\title{
Aplicações de inteligência artificial na determinação de parâmetros de resistência ao cisalhamento do solo: um mapeamento sistemático da literatura
}

Applications of artificial intelligence in determining soil shear strength parameters: a systematic

\author{
literature mapping \\ Aplicaciones de la inteligencia artificial en la determinación de los parámetros de resistencia al \\ cizallamiento del suelo: un mapeo sistemático de la literatura
}

Recebido: 08/12/2021 | Revisado: 14/12/2021 | Aceito: 29/12/2021 | Publicado: 06/01/2022

\author{
Matheus Gomes Carvalho \\ ORCID: https://orcid.org/0000-0002-9708-1139 \\ Universidade Federal do Rio Grande do Norte, Brasil \\ E-mail:mgomesde@gmail.com \\ Eduardo Matthews do Rego Barreto \\ ORCID: https://orcid.org/0000-0003-2818-430X \\ Universidade Federal do Rio Grande do Norte, Brasil \\ E-mail: eduardomatthews@outlook.com \\ José Ailton da Costa Ferreira \\ ORCID: https://orcid.org/0000-0002-8116-3869 \\ Universidade Federal do Rio Grande do Norte, Brasil \\ E-mail: frreirailton@gmail.com \\ Fagner Alexandre Nunes de França \\ ORCID: https://orcid.org/0000-0002-8113-622X \\ Universidade Federal do Rio Grande do Norte, Brasil \\ E-mail: fagnerfranca@ufrn.edu.br \\ Osvaldo de Freitas Neto \\ ORCID: http://orcid.org/0000-0001-9488-4123 \\ Universidade Federal do Rio Grande do Norte, Brasil \\ E-mail: osvaldo.neto@ufrn.br
}

\begin{abstract}
Resumo
A investigação da resistência ao cisalhamento dos solos é tarefa corriqueira em diversos projetos de Engenharia Geotécnica, e.g. fundações, estabilidade de taludes e estruturas de contenção. Ela é usualmente conduzida por meio de ensaios padronizados de campo e/ou laboratório. Nos dois casos, trata-se de uma tarefa que demanda tempo e possui um custo associado a ela. Diversos estudos podem ser encontrados na literatura em que ferramentas de inteligência artificial são usadas como alternativa à execução desses ensaios. Este artigo apresenta um mapeamento sistemático da literatura sobre esse assunto. Os tipos de algoritmo e os parâmetros geotécnicos empregados para estimar a resistência ao cisalhamento do solo foram identificados com base nos dados extraídos da literatura. Foi possível listar 17 técnicas aplicadas a diferentes tipos de solo. Os resultados desses estudos encontram-se de acordo com dados obtidos por meio de ensaios geotécnicos reais, de laboratório e de campo. Isso demonstra o potencial de uso de inteligência artificial para estimar a resistência ao cisalhamento dos solos.
\end{abstract}

Palavras-chave: Mapeamento sistemático; Resistência ao cisalhamento; Inteligência artificial.

\begin{abstract}
The investigation of soils shear strength is necessary in many Geotechnical Engineering applications, e.g. foundations, slope stability and retaining walls design. It is usually conducted by means of field and/or laboratory standard tests. In both cases, they are time-consuming and require specialized personnel to be performed. Several studies are found in the literature in which artificial intelligence tools are used as an alternative to those tests. This paper presents a systematic mapping review on this subject, in which algorithms types and geotechnical parameters needed to estimate soils shear strength are identified, based on data extracted from the literature. It was possible to list 17 techniques applied to different soil types. The results from these studies have been in good agreement with data from real laboratory and/or field tests. This demonstrates the potential of application of artificial intelligence to estimate soils shear strength.

Keywords: Systematic mapping; Shear strength; Artificial intelligence.
\end{abstract}

\section{Resumen}

La investigación de la resistencia al corte de los suelos es una tarea común en varios proyectos de ingeniería geotécnica, por ejemplo, cimentaciones, estabilidad de taludes y estructuras de contención. Por lo general, se lleva a cabo mediante 
pruebas estandarizadas de campo y / o laboratorio. En ambos casos, es una tarea que demanda tiempo y tiene un costo asociado. En la literatura se pueden encontrar varios estudios en los que se utilizan herramientas de inteligencia artificial como alternativa a la realización de estas pruebas. Este artículo presenta un mapeo sistemático de la literatura sobre este tema. Los tipos de algoritmos y parámetros geotécnicos utilizados para estimar la resistencia al corte del suelo se identificaron con base en datos extraídos de la literatura. Fue posible enumerar 17 técnicas aplicadas a diferentes tipos de suelo. Los resultados de estos estudios están de acuerdo con los datos obtenidos a través de pruebas geotécnicas, de laboratorio y de campo reales. Esto demuestra el potencial de usar inteligencia artificial para estimar la resistencia al corte de los suelos.

Palabras clave: Mapeo sistemático; Resistencia al corte; Inteligencia artificial.

\section{Introdução}

A investigação da resistência ao cisalhamento dos solos é parte fundamental no desenvolvimento de empreendimentos geotécnicos, pois, como expõem Terzaghi e Peck (1948), em todos os problemas que envolvem tensões do terreno, capacidade de carga de fundações e estabilidade de taludes, a avaliação da resistência ao cisalhamento do solo em questão é essencial. Assim, é possível dizer que a determinação precisa e confiável dos parâmetros de resistência ao cisalhamento dos solos é de extrema importância para a prática de engenharia.

Terzaghi e Peck (1948) mencionam que, na mecânica dos solos, a representação mais usual do comportamento de ruptura dos solos saturados é feita utilizando-se o critério de Mohr-Coulomb. Ele assume que a ruptura ocorre quando a combinação das tensões normais e cisalhantes $(\sigma, \tau)$ no plano de ruptura é tal que a tensão de cisalhamento é máxima (teoria de Mohr) e que a envoltória dos círculos representativos dos estados de tensões na ruptura, encontrada experimentalmente, é uma reta cuja formulação (Equação 1) é conhecida como equação de Coulomb (Gerscovich, 2010).

$$
\tau=c+\sigma \cdot \tan \varphi
$$

Onde: $\tau$ - resistência ao cisalhamento; $\sigma$ - tensão normal no plano; c - coesão; $\varphi$ - ângulo de atrito interno.

A Equação (1) apresenta a resistência ao cisalhamento " $\tau$ ” variando em função da tensão normal " $\sigma$ " a partir dos parâmetros empíricos c (coesão) e $\varphi$ (ângulo de atrito interno). Pode-se determinar esses parâmetros através de ensaios de campo e laboratório, sendo os ensaios de cisalhamento direto e de compressão triaxial os mais comuns em laboratório (Mollahasani et al., 2011). Durante o ensaio, quando os valores de poropressão " $u_{w}$ " são medidos, a resistência ao cisalhamento pode ser expressa em termos de tensões efetivas, resultando na Equação (2).

$$
\tau=c^{\prime}+\left(\sigma-u_{w}\right) \cdot \tan \varphi^{\prime}
$$

Onde: $\tau$ - resistência ao cisalhamento; $\sigma$ - tensão normal no plano; c - coesão; $\varphi$ - ângulo de atrito interno; $u_{w}$ poropressão.

Vanapalli et al. (1996) afirmam que as análises de estabilidade são, usualmente, baseadas em parâmetros de resistência ao cisalhamento de solos saturados. No entanto, a maioria dos solos encontrados na prática de engenharia apresentam-se em estado não saturado, fazendo com que esse tipo de análise seja considerado conservador por não considerar os efeitos de sucção no solo. Dessa maneira, a diferença no comportamento de solos saturados em relação aos não saturados pode ser atribuída, principalmente, a duas situações. A primeira diz respeito aos solos saturados apresentarem duas fases, sólida e líquida, representados por solo e água, e os não saturados apresentarem quatro fases: sólida, líquida, gasosa e membrana contrátil. As fases gasosa e membrana contrátil são referentes ao ar e a interface ar-água, respectivamente (Fredlund \& Rahardjo, 1993). A segunda situação é relativa ao efeito da sucção nos solos não saturados, relacionada a capacidade de retenção de água no solo e 
definida como a diferença entre a pressão de ar e a pressão de água no solo $\left(u_{a}-u_{w}\right)$. Existem diversas equações utilizadas para interpretar o comportamento de resistência ao cisalhamento de solos não saturados, duas das quais são bastante conhecidas. A primeira publicada por Bishop (1959, apud Garven, 2009) (Equação 3) e a segunda apresentada por Fredlund, Morgenstern e Widger (1978, apud Garven, 2009) (Equação 4).

$$
\begin{aligned}
\tau & =c^{\prime}+\left(\sigma-u_{a}\right) \tan \varphi^{\prime}+\chi\left(u_{a}-u_{w}\right) \\
\tau & =c^{\prime}+\left(\sigma-u_{a}\right) \tan \varphi^{\prime}+\left(u_{a}-u_{w}\right) \tan \varphi^{b}
\end{aligned}
$$

Onde: $\tau$ - resistência ao cisalhamento; $\left(\sigma-u_{a}\right)$ - tensão efetiva; $c^{\prime}$ - coesão efetiva; $\varphi^{\prime}$ - ângulo de atrito interno efetivo; $\left(u_{a}-u_{w}\right)$ - sucção; $\chi$ - parâmetro relacionado ao grau de saturação; $\varphi^{b}$ - ângulo de atrito relativo à sucção.

A resistência ao cisalhamento de um solo pode ser determinada por diferentes métodos, com aplicações em campo ou em laboratório. Em relação aos ensaios de laboratório, os mais usuais são conhecidos como cisalhamento direto e compressão triaxial. Como apresenta Gerscovich (2010), o ensaio de cisalhamento direto consiste na imposição de um plano de ruptura em uma amostra prismática de solo, podendo representar a condição de campo da Figura 1.

Figura 1 - Representação da condição de campo.

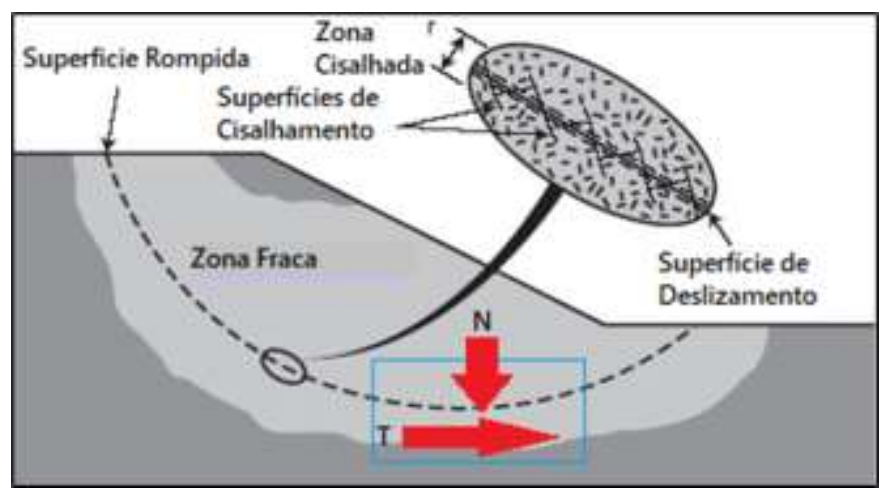

Fonte: Leroueil (2001).

No procedimento de ensaio, utiliza-se uma caixa bipartida, na qual o corpo de prova se encontra sob ação inicial de uma força vertical $(\mathrm{N})$, cuja metade superior possa ser deslocada horizontalmente pela aplicação de uma força tangencial (T). Assim, promove-se o movimento relativo horizontal entre a metade superior e a metade inferior do corpo de prova. A divisão das forças $\mathrm{N}$ e T pela área da seção transversal do corpo de prova resulta nas tensões normal e de cisalhamento, respectivamente, no plano horizontal. As especificações para os componentes do equipamento e procedimentos de realização do ensaio estão expostas nas normas ASTM D3080-11 (American Society for Testing and Materials [ASTM], 2020a), AASHTO T 236-08 (American Association of State and Highway Trasportation Officials [AASHTO], 2008) e BS 1377-7 (British Standards Institution [BSI], 1990).

Outro ensaio comumente utilizado na investigação da resistência ao cisalhamento dos solos é, como descrito em Terzaghi e Peck (1948), o ensaio de compressão triaxial. Na sua montagem convencional, ele é realizado em um corpo de prova cilíndrico que, envolto por uma membrana de borracha e colocado em câmara de ensaio preenchida com água, sofre a ação de uma tensão confinante em todas as direções (estado hidrostático de tensões) seguido por um carregamento axial de compressão independente. A Figura 2 representa um esquema da câmara de ensaio triaxial. 
Figura 2 - Esquema do ensaio de compressão triaxial.

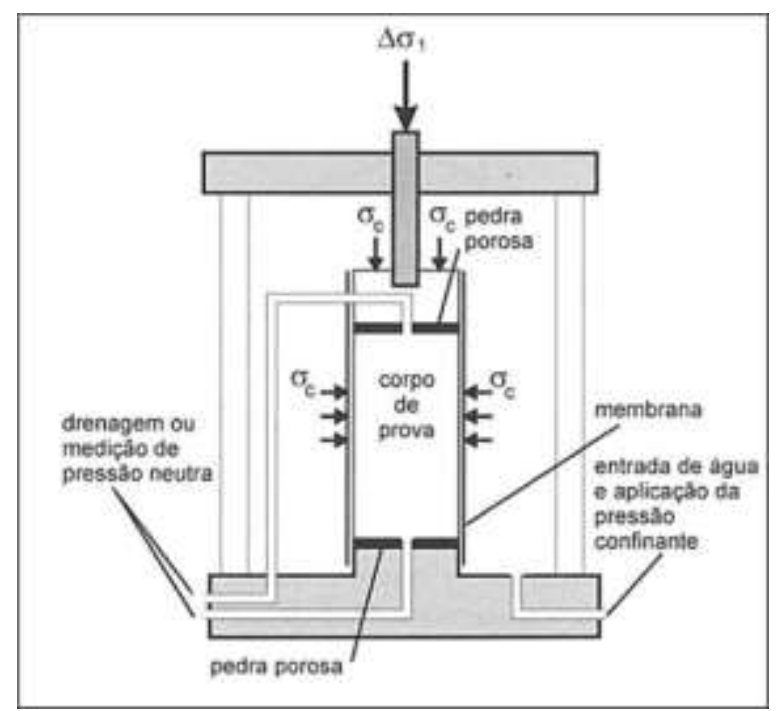

Fonte: Pinto (2006).

Pode-se dividir esse ensaio em duas etapas. Na etapa inicial, chamada de adensamento, a drenagem pode ser permitida, situação em que a pressão neutra é dissipada e o corpo de prova é adensado sob a tensão confinante. Este tipo de ensaio é chamado de adensado. Quando não se permite a drenagem e a consequente dissipação de pressão neutra no corpo de prova, o ensaio é determinado como não adensado. Na segunda fase, chamada de cisalhamento, novamente, a drenagem pode ser permitida, ensaio drenado, ou não permitida, ensaio não drenado. As combinações dos modos de execução da primeira e segunda etapa produzem convencionalmente os ensaios Não Adensado e Não Drenado ou "UU" (ASTM D2850-15), Adensado e Drenado ou "CD" (ASTM D7181-20) e o ensaio Adensado e Não Drenado ou "CU” (ASTM D4767-20) (ASTM, 2015; ASTM, 2020b; ASTM. 2020c).

A determinação dos parâmetros de resistência dá-se usualmente para cada novo projeto e situação encontrada. Utilizamse equipamentos que demandam energia elétrica, manutenções periódicas e pessoal operacional capacitado. Além disso, em determinadas situações, a execução dos referidos ensaios pode ter um custo elevado, como em projetos com restrições de orçamento e tempo, quando a disponibilidade de equipamento para realização dos ensaios é limitada ou quando o acesso aos laboratórios é restrito na região em questão.

As limitações apresentadas motivaram vários autores a desenvolver soluções numéricas para estimar os valores dos parâmetros de resistência, por meio da relação entre eles e outras propriedades dos solos. Contudo, essa metodologia possui uma precisão limitada, pois o comportamento de resistência ao cisalhamento dos solos é afetado por diversos fatores que, por sua vez, podem apresentar uma relação não-linear com a resistência ao cisalhamento. Em razão dessas dificuldades, auxiliados pelos avanços observados em ciência da computação nas últimas décadas, diversos autores têm utilizado ferramentas de inteligência artificial para construção de modelos de previsão dos parâmetros de resistência ao cisalhamento dos solos (Kanungo, Sharma \& Pain, 2014; Bui, Hoang \& Nhu, 2018).

A Inteligência artificial (I.A.) é um ramo da ciência da computação que estuda o desenvolvimento de agentes inteligentes. Pode-se considerar agente inteligente algo ou alguém que, em um determinado ambiente, pratica uma ação que seja apropriada às suas circunstâncias e objetivos, sendo esta ação derivada do que se percebe no ambiente. $\mathrm{O}$ objetivo científico central da inteligência artificial é entender os princípios que regem os comportamentos inteligentes em sistemas naturais ou artificiais, sendo sua principal hipótese a ideia de que o raciocínio por trás destes comportamentos é computacional (Poole, Mackworth \& Goebel, 1998). 
Um dos campos mais explorados da inteligência artificial é o aprendizado de máquina, que se define como o domínio centrado na construção de algoritmos capazes de "aprender", ou seja, tomar decisões e/ou fazer previsões a partir de dados fornecidos (Kohavi \& Provost, 1998). Esses algoritmos são os mais utilizados em resolução de problemas de engenharia por sua capacidade de estabelecer padrões que correlacionem os dados, através de exemplos, mesmo sem um conhecimento prévio das relações entre eles, em contraste com os modelos tradicionais desenvolvidos a partir da natureza das relações físicas entre partículas. Dentre as diversas técnicas de aprendizado de máquina existentes, duas das mais aplicadas na resolução de problemas não-lineares em engenharia são redes neurais artificiais e algoritmos genéticos (Fatehnia \& Amirinia, 2018).

Redes neurais artificiais são ferramentas de modelagem computacional desenvolvidas com base no que se sabe sobre o funcionamento do cérebro humano, a partir de uma abstração de como se acredita que os neurônios processem informação. Esses algoritmos são formados por redes de neurônios artificiais, chamados de nós ou elementos de processamento do modelo matemático, que são distribuídos em diferentes camadas (Basheer \& Hajmeer, 2000).

Numa rede neural típica existem três tipos de camadas: a camada de nós de entrada de dados, a camada de nós de saída e as camadas escondidas, que ficam entre as duas primeiras. Todos os nós em uma camada são conectados a todos os nós da camada sucessiva e a essa conexão é atribuído um ponderador (Shahin, Jaksa \& Maier, 2009). A estrutura de uma rede neural artificial típica está representada na Figura 3. O processo de aprendizado, ou treinamento, de uma rede neural artificial consiste, portanto, na iteração dos valores dos ponderadores com o objetivo de se conceber um modelo capaz de apresentar valores de saída satisfatórios quando alimentados com novos valores de entrada (Das, 2013). Exemplos de aplicação de redes neurais na engenharia geotécnica são mostrados nos estudos desenvolvidos por Holanda et al. (2021) e Pessoa et al. (2021).

Figura 3 - Estrutura de uma rede neural artificial típica

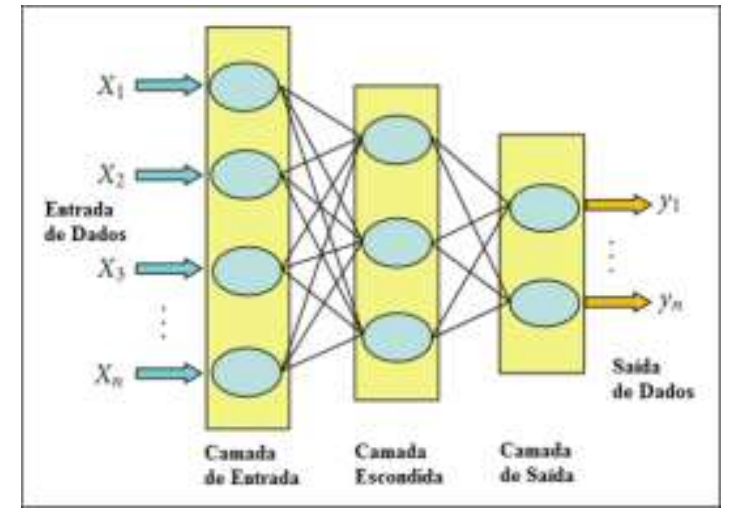

Fonte: Shahin, Jaksa e Maier (2009).

A computação evolucionária, por sua vez, é um subcampo da inteligência artificial que utiliza algoritmos baseados em mecanismos evolutivos observados na natureza para geração de soluções aplicáveis a determinado problema. O funcionamento básico dos algoritmos evolucionários consiste na geração de um conjunto de soluções aleatórias durante a fase de inicialização, que são avaliadas por uma função de aptidão (fitness) selecionando os melhores candidatos que, por sua vez, servirão de base para as próximas gerações de soluções. Esse processo é repetido até que se observe soluções que atendam a critérios especificados (Eiben \& Smith, 2015).

A forma como as soluções são avaliadas e como se dá a variação de uma geração para outra depende do problema a ser explorado e do tipo de algoritmo utilizado. Em engenharia civil, os algoritmos evolucionários são aplicados, principalmente, na resolução de problemas de otimização complexos. De acordo com Lu, Chen e Zheng (2012), alguns dos algoritmos evolucionários mais empregados na engenharia civil são: algoritmos e programação genética (baseados no princípio 
evolucionário de sobrevivência do mais adaptado) e sistemas imunes artificiais (baseados nos sistemas imunológicos dos seres vivos).

Diante da problemática apresentada, este artigo tem o objetivo de realizar um mapeamento sistemático de publicações em que são aplicadas técnicas de inteligência artificial para determinação dos parâmetros de resistência ao cisalhamento do solo. Assim, é possível estabelecer uma visão geral sobre a área e servir de base para o desenvolvimento de novos trabalhos a partir da consideração dos métodos utilizados e da avaliação de seus resultados.

\section{Metodologia}

Este trabalho utilizou uma adaptação do método apresentado por Kitchenham e Charters (2007) para Revisões Sistemáticas da Literatura (Systematic Literature Review), RSL, em Engenharia de Software. Embora, devido ao caráter mais amplo e exploratório de seu objetivo geral (questão de pesquisa), como exposto por Petersen, Vakkalanka e Kuzniarz (2015), o presente artigo seria melhor classificado dentro dos Estudos de Mapeamento Sistemático (Systematic Mapping Studies).

Como ponto de partida, foram definidas as questões de pesquisa, derivadas dos objetivos estabelecidos para o trabalho, apontadas a seguir.

Questão 1 - Como ferramentas de inteligência artificial têm sido utilizadas na investigação (determinação de parâmetros) da resistência ao cisalhamento dos solos?

Questão 2 - Quais ferramentas de inteligência artificial têm sido utilizadas na investigação da resistência ao cisalhamento dos solos?

Questão 3 - Qual a qualidade dos resultados encontrados em comparação com os resultados de métodos convencionais?

Posteriormente, como parte da estratégia de pesquisa, a metodologia PICO (Population, Intervention, Comparison and Outcomes), sugerida em Kitchenham e Charters (2007), foi utilizada na definição da string de busca. A expressão "Soil Shear Strenght" foi utilizada como representativa da População e a expressão "Artificial Intelligence" da Intervenção. Os termos relativos à Comparação e Efeitos foram excluídos por representarem uma restrição nos resultados de busca, o que seria desejável na elaboração de uma RSL, mas não neste caso.

Os resultados foram obtidos por meio do Portal Periódicos CAPES, no modo busca avançada, com as opções "Qualquer" e "Contém" selecionadas, restringindo o resultado de busca aos trabalhos publicados entre os anos de 2008 e 2018. Com os resultados retornados pela busca, primeiramente, utilizou-se o critério de avaliação do título e resumo dos trabalhos para exclusão de artigos não desejados e/ou repetidos, resultando na seleção de publicações que, posteriormente, foram analisadas pela leitura do completo teor. Os critérios utilizados na análise de exclusão dos artigos estão listados a seguir.

- Exclusão de trabalhos por incompatibilidade com o tema "Resistência ao cisalhamento dos solos" (e.g. resistência a compressão do solo, resistência ao cisalhamento de estruturas de fundação, resistência ao cisalhamento de geossintéticos, etc.).

- Exclusão de trabalhos que utilizavam apenas métodos estatísticos clássicos, como regressões lineares e equações semiempíricas, quando não definidas pelo autor como uma aplicação de inteligência artificial. Para a aplicação deste critério observou-se a seleção de palavras chave e a revisão da literatura apresentada.

- Exclusão de trabalhos referentes à área de agricultura, cuja investigação era relacionada a resistência ao cisalhamento superficial dos solos.

Vale ressaltar que, para Kitchenham e Charters (2007), o processo de desenvolvimento da estratégia de pesquisa é, usualmente, iterativo, de modo que foram testadas outras combinações de strings de busca, retornando diferentes quantidades de resultados. Entretanto, após a aplicação dos critérios de exclusão observou-se uma quantidade menor de artigos em relação à estratégia adotada. 
Como a quantidade de trabalhos resultantes da busca e aplicação dos critérios de exclusão foi considerada pequena, estudos adicionais foram selecionados utilizando a técnica snowball de amostragem, em que artigos que citam ou são citados pelos trabalhos selecionados são escolhidos e avaliados segundo os mesmos critérios de exclusão. Para o cruzamento de referências, foram utilizadas as plataformas Google Scholar, ResearchGate e Scopus.

Com o número final de publicações selecionadas, desenvolveu-se uma estratégia de extração de dados, que consiste em um formulário no qual os campos a serem preenchidos contemplam as informações que, após analisadas e classificadas, serão apresentadas como resultado da revisão em forma de gráficos e quadros. A estratégia de extração de dados do presente trabalho foi elaborada com base nos campos da tabela apresentada por Fatehnia e Amirinia (2018).

\section{Resultados e Discussão}

Incialmente, foram selecionados 24 artigos, dentre os quais 10 permaneceram após a análise de títulos e resumos e 6 artigos foram escolhidos após a avaliação de conteúdo completo. Em adição, houve a escolha de outros 12 artigos pela técnica snowball, totalizando assim 18 publicações selecionadas para este estudo. A Figura 4 indica a distribuição de artigos em relação ao ano de publicação.

Figura 4 - Quantidade de artigos selecionados por ano de publicação.

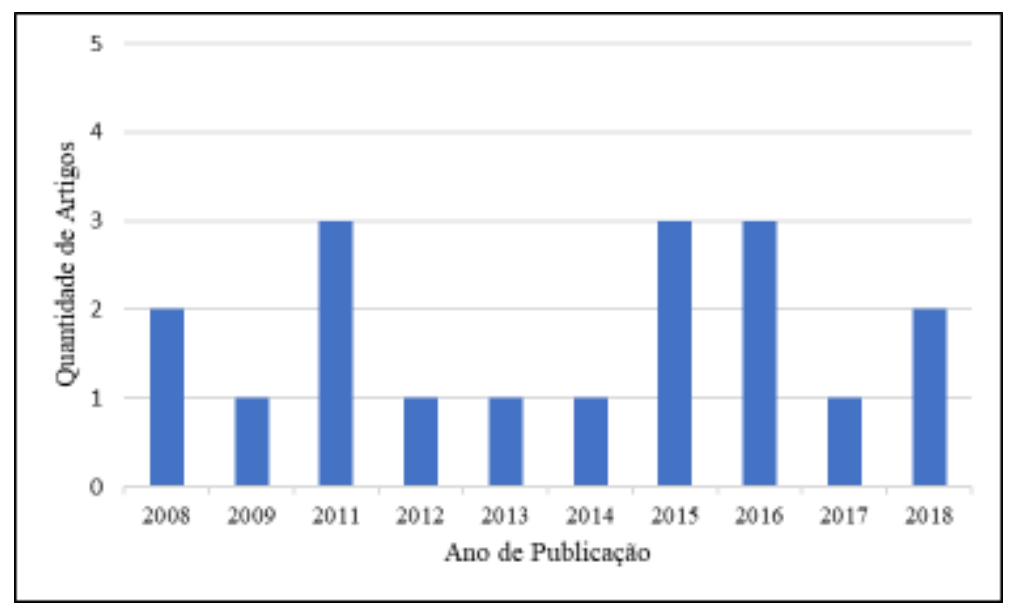

Fonte: Autores (2021).

As previsões obtidas pelos modelos a partir de novos dados de entrada são limitadas pela representatividade dos dados utilizados na etapa de treinamento. Um dos fatores que podem ser utilizados na consideração da representatividade é a origem da base de dados. Nos trabalhos estudados, observaram-se os seguintes tipos: dados retirados da literatura e dados advindos de amostras coletadas em regiões que coincidem com os países de origem dos artigos. Dessa maneira, a distribuição de artigos por país de origem está disposta na Figura 5. 
Figura 5 - Quantidade de artigos selecionados por ano de publicação.

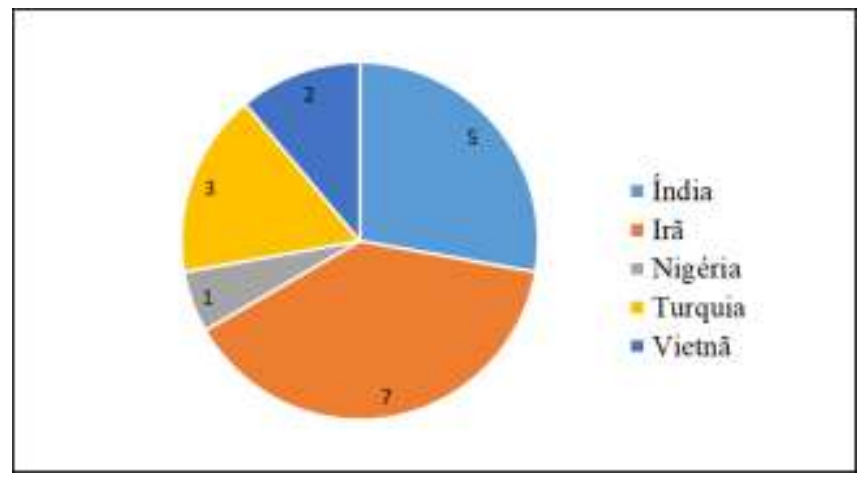

Fonte: Autores (2021).

Na Figura 6, tem-se a representação das técnicas empregadas pelos autores nos artigos selecionados. Vale salientar que em alguns artigos utilizaram-se mais de uma técnica. Os 17 tipos de algoritmo utilizados encontram-se listados na Tabela 1.

Figura 6 - Números de utilizações das técnicas nas publicações.

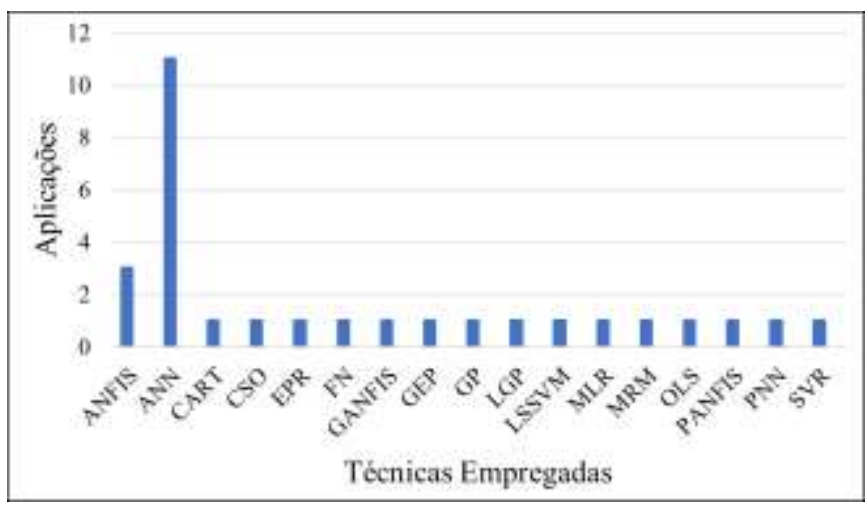

Fonte: Autores (2021).

Tabela 1 - Técnicas empregadas nos artigos.

\begin{tabular}{c|c}
\hline Abreviação & Técnica Empregada \\
\hline ANFIS & Adaptive Neuro Fuzzy Inference System \\
ANN & Artificial Neural Network \\
CART & Classification And Regression Trees \\
CSO & Cuckoo Search Optimization \\
EPR & Evolutionary Polynomial Regression \\
FN & Functional Networks \\
GANFIS & Genetic Algorithm - Adaptive Network Based Fuzzy Inference System \\
GEP & Gene Expression Programming \\
GP & Genetic Programming \\
LGP & Linear Genetic Programming \\
LSSVM & Least Squares Support Vector Machine \\
MLR & Multiple Linear Regression \\
MRM & Multiple Regression Models \\
OLS & Orthogonal Least Squares \\
PANFIS & Particle Swarm Optimization - Adaptive Network Based Fuzzy Inference System \\
PNN & Probabilistic Neural Network \\
SVR & Suport Vector Regression \\
\hline
\end{tabular}

Fonte: Autores (2021). 
A escolha das variáveis de entrada é parte fundamental do desenvolvimento dos modelos, visto que esses fatores são considerados influenciadores do comportamento resistente do solo ao cisalhamento. Alguns dos autores selecionaram as variáveis de entrada a partir de revisão da literatura, enquanto que outros utilizaram as propriedades índices do solo (do original - Soil Index Properties). As características do solo utilizados como variáveis de entrada encontram-se listadas na Tabela 2, enquanto que na Tabela 3 tem-se as variáveis de saída, que representam os parâmetros de resistência ao cisalhamento do solo a serem determinados.

Tabela 2 - Variáveis de entrada dos modelos.

\begin{tabular}{|c|c|c|}
\hline Simbol. & Variável de entrada & Termo Original \\
\hline $\mathrm{H}$ & Profundidade da amostra & Sample Depth \\
\hline $\mathrm{A} \%$ & Percentual de Areia & Sand Percentage \\
\hline $\mathrm{L} \%$ & Percentual de Loam & Loam Percentage \\
\hline $\mathrm{C} \%$ & Percentual de Argila & Clay Percentage \\
\hline $\mathrm{TU}$ & Teor de Umidade & Moisture Content \\
\hline PES & Peso Específico Saturado & Wet Density of Soil \\
\hline GE & Gravidade Específica & Specific Gravity \\
\hline LL & Limite de Liquidez & Liquid Limit \\
\hline LP & Limite de Plasticidade & Plastic Limit \\
\hline IP & Índice de Plasticidade & Plastic Index \\
\hline IL & Índice de Liquidez & Liquid Index \\
\hline $\mathrm{P} \%$ & Percentual de Pedregulho & Gravel Percentage \\
\hline ST\% & Percentual de Silte & Silte Percentage \\
\hline PSC & Peso Específico Seco & Dry Density \\
\hline $200 \%$ & Percentual passante peneira $\mathrm{N}^{\circ} 200$ & Percentage passing $N^{\circ} 200$ \\
\hline $40 \%$ & Percentual passante peneira $\mathrm{N}^{\circ} 40$ & Percentage passing $N^{\circ} 40$ \\
\hline $4 \%$ & Percentual passante peneira $\mathrm{N}^{\circ} 4$ & Percentage passing $N^{\circ} 4$ \\
\hline $\mathrm{D}$ & Densidade & Density \\
\hline $\mathrm{F} \%$ & Percentual de Finos & Fine-grained Content \\
\hline $\mathrm{G} \%$ & Percentual Grosseiro & Coarse-grained Content \\
\hline D10 & Diâmetro em que $10 \%$ da massa de solo passa na peneira & Grain size for which $10 \%$ of the sample was finer \\
\hline D30 & Diâmetro em que $30 \%$ da massa de solo passa na peneira & Grain size for which $30 \%$ of the sample was finer \\
\hline D60 & Diâmetro em que $60 \%$ da massa de solo passa na peneira & Grain size for which $60 \%$ of the sample was finer \\
\hline $\mathrm{Cu}$ & Coeficiente de uniformidade & Coefficient of Uniformity \\
\hline $\mathrm{Cc}$ & Coeficiente de Curvatura & Coefficient of Curvature \\
\hline SUW & Peso Específico & Soil Unit Weight \\
\hline SDUW & Densidade Aparente Seca & Soil Dry Unit Weight \\
\hline TNL & Tensão Normal Liquida & Net Normal Stress \\
\hline SM & Sucção Matricial & Matric Suction \\
\hline$c^{\prime}$ & Coesão Efetiva & Effective Cohesion \\
\hline$\Phi$ & Ângulo de Resistência ao Atrito & Angle of Frictional resistance \\
\hline PEN & Peso Específico Natural & Bulk Density \\
\hline TC & Taxa de Cisalhamento & Shearing Rate \\
\hline $\mathrm{A} \%$ & Porcentagem de Argila & Clay Fraction \\
\hline DPI & Desvio da linha A no gráfico de plasticidade & The deviation from the A-line in classification chart \\
\hline $\mathrm{DR}$ & Densidade Relativa & Relative Density \\
\hline $\mathrm{DF}$ & Dimensão Fractal & Fractal Dimension \\
\hline $\mathrm{R}$ & Regularidade & Regularity \\
\hline
\end{tabular}

${ }^{1}$ Termo genérico aplicado a solos férteis, contendo matéria orgânica, areia, silte e argila. Fonte: Autores (2021). 
Tabela 3 - Variáveis de saída dos modelos.

\begin{tabular}{l|l|l}
\hline Simbol. & Variável de saída & Termo Original \\
\hline Ss & Resistência ao Cisalhamento & Shear Strength \\
$\mathrm{c}$, c' & Coesão, Coesão Efetiva & Cohesion, Effective Cohesion \\
$\phi, \phi$ & Ângulo de Atrito, Ângulo de Atrito Efetivo & Friction Angle, Effective Friction Angle \\
$\mathrm{Su}$ & Resistência ao Cisalhamento Não Saturada & Unsaturated Shear Strength \\
\hline
\end{tabular}

Fonte: Autores (2021)

Na maioria das publicações selecionadas foi feita uma avaliação estatística de correlação entre as variáveis de entrada e saída com o objetivo de identificar as variáveis mais representativas. Esses resultados variam de modelo para modelo, de modo que os fatores mais utilizados foram: índice de plasticidade (IP), limite de liquidez (LL), distribuição granulométrica e peso específico.

Observa-se que as variáveis de entrada utilizadas correspondem a parâmetros que são obtidos via ensaios de campo e de laboratório, além do que, alguns deles, como os limites de Atterberg, são usualmente determinados a partir de métodos que estão sujeitos a interferência do operador no resultado. Isso pode limitar a aplicação dos modelos no que diz respeito ao acesso a equipamentos, disponibilidade de tempo e confiabilidade dos resultados.

Ademais, deve-se considerar os aspectos que limitaram os resultados deste trabalho, como a string e a plataforma de busca escolhidas, o fato de a seleção dos artigos ter sido feita por apenas um autor e a estratégia de extração de dados adotada. Os resultados obtidos apresentam um conjunto de algoritmos de inteligência artificial, aplicados a dados de diferentes tipos de solo, cujo desempenho na determinação de parâmetros de resistência ao cisalhamento, na maioria dos casos, foi considerado satisfatória pela avaliação de índices estatísticos como coeficiente de correlação, raiz do erro quadrático médio e erro médio absoluto.

A Tabela 4 apresenta as informações extraídas dos artigos selecionados com a aplicação da estratégia de extração de dados. Ela representa um resumo dos fatores que podem ser analisados no caso de reprodução dos métodos descritos nos artigos. Deve-se considerar as limitações de replicabilidade relativas à representatividade das amostras nos bancos de dados utilizados nos modelos e a disponibilidade dos equipamentos necessários para obtenção dos parâmetros de entrada. Contudo, observa-se na variabilidade dos conjuntos de dados de entrada escolhidos, a possibilidade de desenvolvimento de aplicações práticas utilizando-se dos modelos nos quais a obtenção das variáveis de entrada seja viável, de acordo com a situação de projeto encontrada. 
Tabela 4 - Dados extraídos dos artigos selecionados.

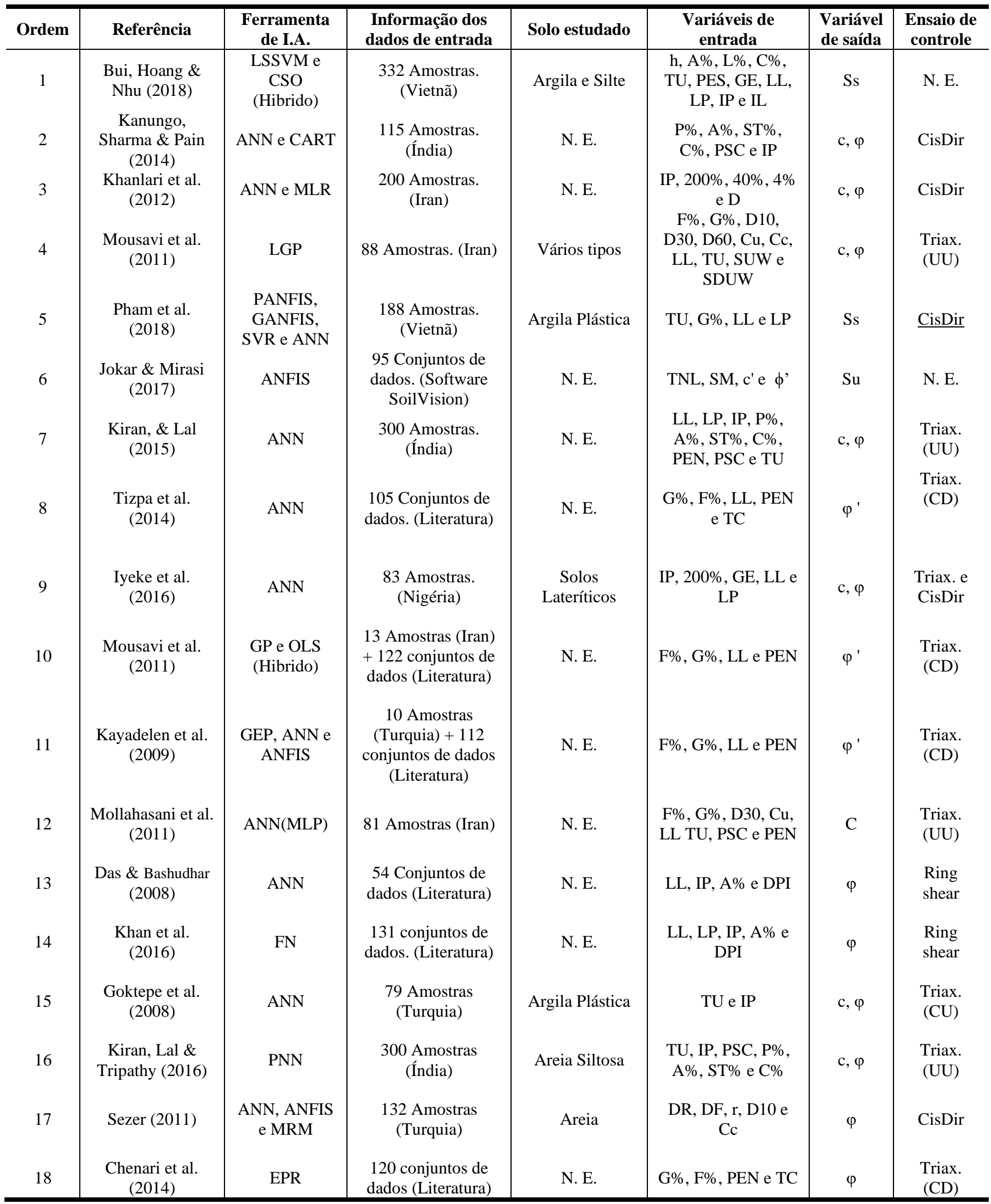

Legenda: CisDir - Ensaio de Cisalhamento Direto, Triax. - Ensaio de Cisalhamento Triaxial, N.E - Não Especificado. Fonte: Autores (2021). 


\section{Conclusão}

Este estudo analisou 18 artigos científicos nos quais foram aplicadas 17 diferentes técnicas de inteligência artificial com o objetivo de estimar parâmetros de resistência ao cisalhamento dos solos. A maior parte dessas técnicas está relacionada às redes neurais artificiais e aos algoritmos evolucionários. Os trabalhos avaliados utilizaram de diversos conjuntos de dados de diferentes tipos de solo, nos quais as variáveis de entrada exploradas com maior frequência foram: índice de plasticidade (IP), limite de liquidez (LL), distribuição granulométrica e peso específico dos solos. É importante salientar a ausência de artigos brasileiros nesse conjunto de dados, demonstrando a necessidade de desenvolvimento na área.

Os resultados apresentados demonstram o emprego satisfatório de diversos algoritmos de inteligência artificial na investigação do problema em questão e incentivam a produção de novos estudos com foco em abordagens alternativas, trabalhos que explorem as variadas aplicações em geotecnia de técnicas computacionais específicas e o desenvolvimento de metodologias que possibilitem a utilização do potencial de recentes avanços das ferramentas computacionais nos projetos de engenharia. Assim, a discussão exposta direciona novas pesquisas à utilização de redes neurais artificiais, uma vez que dentre os artigos estudados é a mais utilizada e produz resultados satisfatórios a partir diferentes conjuntos de variáveis de entrada.

\section{Agradecimentos}

O presente trabalho foi realizado com apoio da Coordenação de Aperfeiçoamento de Pessoal de Nível Superior Brasil (CAPES) - Código de Financiamento 001.

\section{Referências}

American Association of State and Highway Trasportation Officials (2008). AASHTO T 236-08: Standard Method of Test for Direct Shear Test of Soils Under Consolidated Drained Conditions. Washington, DC.

American Society for Testing and Materials (2020a). ASTM D 3080-11: Standard Method for Direct Shear Test of Soils Under Consolidated Drained Conditions. Filadélfia: ASTM International.

American Society for Testing and Materials (2015). ASTM D 2850-15: Standard Test Method for Unconsolidated-Undrained Triaxial Compression Test on Cohesive Soils. Filadélfia: ASTM International.

American Society for Testing and Materials (2020b). ASTM D 7181-20: Standard Test Method for Consolidated Drained Triaxial Compression Test for Soils. Filadélfia: ASTM International.

American Society for Testing and Materials (2020c). ASTM D 4767-20: Standard Test Method for Consolidated Undrained Triaxial Compression Test for Cohesive Soils. Filadélfia: ASTM International.

British Standards Institution (1990). BS 1377-7: Methods of test for Soils for civil engineering purposes - Part 7: Shear strength tests (totals stress). London: Britsh Standards Institution.

Basheer, I. A. \& Hajmeer, M. (2000). Artificial neural networks: fundamentals, computing, design, and application. Journal Of Microbiological Methods, [s.1.], 43(1), 3-31.

Bui, D. T., Hoang, N. \& Nhu, V. (2018). A swarm intelligence-based machine learning approach for predicting soil shear strength for road construction: a case study at Trung Luong National Expressway Project (Vietnam). Engineering With Computers, [s.1.], 1-11.

CAPES - Coordenação de Aperfeiçoamento de Pessoal de Nível Superior. Portal de Periódicos Capes. Brasil. Recuperado de http://www.periodicos.capes.gov.br/.

Chenari, R. J., Tizpa, P., Rad, M. R. G., Machado, S. L. \& Fard, M. K. (2014). The use of index parameters to predict soil geotechnical properties. Arabian Journal Of Geosciences, [s.1.], 8(7), 4907-4919.

Das, S. K. (2013) Artificial Neural Networks in Geotechnical Engineering: Modeling and Application Issues: Metaheuristics in Water. Geotechnical And Transport Engineering, Londres, p.231-270.

Das, S. K. \& Bashudhar, P. K. (2008). Prediction of residual friction angle of clays using artificial neural network. Engineering Geology, [s.1.], 100(3-4), 142145 .

Eiben, A. E. \& Smith, J. (2015). From evolutionary computation to the evolution of things. Nature, [s.1.], 521(7553), 476-48. 
Fatehnia, M. \& Amirinia, G. (2018). A review of Genetic Programming and Artificial Neural Network applications in pile foundations. International Journal Of Geo-engineering, [s.1.], 9(1), 1-20.

Fredlund, D. G. \& Rahardjo, H. (1993). Soil Mechanics for Unsaturated Soils. New York: John Wiley \& Sons, Inc, 507 p.

Garven, E. A. (2009). Review of the empirical equations for predicting the shear strength of unsaturated soils. Tese de Doutorado Curso de Engenharia Civil, Faculdade de Engenharia, Universidade de Otawa, Otawa.

Gerscovich, D M. S. (2010). Resistência ao Cisalhamento. Rio de Janeiro: Feuerj. Recuperado de http://www.eng.uerj.br/ denise/pdf/resistenciacisalhamento.pdf.

Goktepe, A. B., Altun, S., Altintas, G. \& Tan, O. (2008). Shear strength estimation of plastic clays with statistical and neural approaches. Building And Environment, [s.1.], 43(5), 849-860

Holanda, M. J. O., Ferreira, S. R. M., Amorim, S. F., Borges, J. J. S. \& Silva, L. F. (2021). Identification of expansive and collapsible soils in northeastern Brazil from Artificial Neural Networks generated in Pernambuco. Research, Society and Development [s.1.], 10(15), e110101522541. DOI: 10.33448/rsd-v10i15.22541

Iyeke, S. D., Eze, E. O., Ehiorobo, J. O. \& Osuji, S.O. (2016). Estimation Of Shear Strength Parameters Of Lateritic Soils Using Artificial Neural Network. Nigerian Journal Of Technology, [s.1.], 35(2), 260-269.

Jokar, M. H. \& Mirasi, S. (2017). Using adaptive neuro-fuzzy inference system for modeling unsaturated soils shear strength. Soft Computing, [s.1.], 22(13), 4493-4510.

Kanungo, D. P., Sharma, S. \& Pain, A. (2014). Artificial Neural Network (ANN) and Regression Tree (CART) applications for the indirect estimation of unsaturated soil shear strength parameters. Frontiers Of Earth Science, [s.1.], 8(3), 439-456.

Kayadelen, C., Gunaydin, O., Fener, M., Demir, A. \& Ozvan, A. (2009). Modeling of the angle of shearing resistance of soils using soft computing systems. Expert Systems With Applications, [s.1.], 36(9), 11814-11826.

Khan, S.Z, Suman, S., Pavani, M. \& Das, S. K. (2016). Prediction of the residual strength of clay using functional networks. Geoscience Frontiers, [s.1.], 7(1), 67-74.

Khanlari, G.R., Heidari, M., Momeni, A. A. \& Abdilor, Y. (2012). Prediction of shear strength parameters of soils using artificial neural networks and multivariate regression methods. Engineering Geology, [s.1.], 131-132, 11-18.

Kiran, S. \& Lal, B. (2015). ANN based Prediction of Shear Strength of Soil from their index properties. International Journal of Earth Sciences and Engineering, [s.1.].

Kiran, S., LAL, B. \& Tripathy, S.S. (2016). Shear Strength Prediction of Soil based on Probabilistic Neural Network. Indian Journal Of Science And Technology, [s.1.], 9(41).

Kitchenham, B. \& Charters., S. (2007). Guidelines for performing systematic literature reviews in software engineering. Technical report. Durham: EBSE.

Kohavi, R. \& Provost, F. (1998). Glossary of Terms. Machine Learning, Boston, 30, 271-274.

Leroueil, S. (2001). Natural slopes and cuts: movement and failure mechanisms. Géotechnique, [s.1.], 51(3), 197-243.

Lu, P., Chen, S. \& Zheng, Y. (2012). Artificial Intelligence in Civil Engineering. Mathematical Problems In Engineering, [s.1.], $2012,1-22$.

Mollahasani, A., Alavi, A. H., Gandomi, A. H. \& Rashed, A. (2011). Nonlinear neural-based modeling of soil cohesion intercept. Ksce Journal Of Civil Engineering, [s.1.], 15(5), 831-840.

Mousavi, S. M., Alavi, A. H., Gandomi, A. H. \& Arab Esmaeili, M. (2011). Formulation of soil angle of shearing resistance using a hybrid GP and OLS method. Engineering With Computers, [s.1.], 29(1), 37-53.

Mousavi, S. M., Alavi, A. H., Gandomi, A. H. \& Mollahasani, A. (2011). Nonlinear genetic-based simulation of soil shear strength parameters. Journal Of Earth System Science, [s.1.], 120(6), 1001-1022.

Pessoa, A. D., Sousa, G. C. L., Araujo, R. C. \& Anjos, G. J. M. (2021). Artificial neural network model for predicting load capacity of driven piles. Research, Society and Development, [s.1.], 10(1), e12210111526. DOI: 10.33448/rsd-v10i1.11526

Petersen, K., Vakkalanka, S. \& Kuzniarz, L. (2015). Guidelines for conducting systematic mapping studies in software engineering: An update. Information And Software Technology, [s.1.], 64, 1-18.

Pham, B. T., Son, L. H., Hoang, T.A., Nguyen, D. M. \& Bui, D, T. (2018). Prediction of shear strength of soft soil using machine learning methods. Catena, [s.1.], 166, 181-191.

Pinto, C. de S. (2006). Curso Básico de Mecânica dos Solos. 3. ed. São Paulo: Oficina de Textos.

Poole, D., Macworth, A. \& Goebel, R. (1998). Computational intelligence: A logical approach. Nova York: Oxford University Press.

Sezer, A. (2011). Simple models for the estimation of shearing resistance angle of uniform sands. Neural Computing And Applications, [s.1.], 22(1), 111-123.

Shahin, M. A., Jaksa, M. B. \& Maier, H. R. (2009). Recent Advances and Future Challenges for Artificial Neural Systems in Geotechnical Engineering Applications. Advances In Artificial Neural Systems, [s.1.], 2009, 1-9.

Terzaghi, K. \& Peck, R. B. (1948). Soil Mechanics in Engineering Practice. 3. ed. Nova York: John Wiley \& Sons Inc. 
Research, Society and Development, v. 11, n. 1, e27711124506, 2022

(CC BY 4.0) | ISSN 2525-3409 | DOI: http://dx.doi.org/10.33448/rsd-v11i1.24506

Tizpa, P., Chenari, R. J. \& Fard, M. K. \& Machado, S. L. (2014). ANN prediction of some geotechnical properties of soil from their index parameters. Arabian Journal Of Geosciences, [s.1.], 8(5), 2911-2920.

Vanapalli, S. K., Fredlund, D. G., Pufahl, D. E. \& Clifton, A. W. (1996). Model for the prediction of shear strength with respect to soil suction. Canadian Geotechnical Journal, [s.1.], 33(3), 379-392. 